\title{
Effects of evidence-based treatment and consent on professional autonomy
}

\author{
C. D. Cannavina, ${ }^{1}$ G. Cannavina, ${ }^{2}$ and T. F. Walsh, ${ }^{3}$
}

\begin{abstract}
Recent high profile cases like Wisheart (Bristol babies) has placed healthcare professional autonomy under both ethical and legal scrutiny, reflected by the growing number of civil cases brought by the public. The aim of this paper is to investigate the effect of evidence-based treatment on the legal requirements of consent, when the treatment transcends the therapeutic boundary. The potential effect on the autonomy of healthcare professionals is also discussed. The study revealed that although consent remains the cornerstone of defence in many areas of therapeutic treatment, it is open to significant legal challenge in the grey area between therapeutic evidence-based treatment and non-evidenced-based procedures. Consent remains the cornerstone of defence for therapeutic procedures. However, it is less certain with innovative treatment or procedures that transcend the therapeutic boundaries and are shown to be non-evidence-based.
\end{abstract}

$\mathrm{M}$ ost definitions of the term 'profession' are descriptive rather than succinct. The professions all have in common the requirement of members to: acquire and maintain specialist knowledge; derive a knowledge-based authority; control access into the profession; control their conduct by professional codes; and be institutionalised and serve the public. ${ }^{1}$ Professions effectively seek to self-regulate and achieve collective autonomy for the institution and individual autonomy for the members. This may be expressed as monopoly for a public service which brings with it professional responsibilities to meet public expectations.

Professions can only achieve autonomy by gaining the public's trust 'credat emptor' (let the buyer trust) as distinct from the market and services to which the maxim 'caveat emptor' (let the buyer beware) generally applies. ${ }^{2}$ Yet in the provision of healthcare services professional autonomy cannot be

${ }^{1}$ Visiting Lecturer (Law and Ethics), ${ }^{2 *}$ Lecturer,

${ }^{3}$ Professor (Head of Department), Department of

Restorative Dentistry, School of Clinical Surgery,

University of Sheffield, Claremont Crescent, Sheffield

S10 2TA

${ }^{*}$ Correspondence to: G. Cannavina

e-mail:g.cannavina@sheffield.ac.uk

REFEREED PAPER

Received 11.06.99; accepted 02.09.99

(C) British Dental Journal 2000; 188: 302-306 either self-sufficient or absolute, as it requires a voluntary suspension of patient autonomy. This suspension is usually conceded on the basis of trust; trust that the health care professional acts always in the best interests of their patients. Professional autonomy therefore carries with it both privileges and responsibilities.

Recent events like the Wisheart case have placed the autonomy of health care professionals under both ethical and legal scrutiny thereby calling into question the professional claim to 'credat emptor.', 3 ' Public concern about such cases is reflected in the growing number of civil cases being brought and the increase in payouts

\section{In brief}

- A loss of patient trust in the health professions has resulted in a reduction in professional autonomy and a rise in litigation and payouts by the courts

- It can be argued that consent and evidence-based treatment (EBT) have become the tools of litigants, with a reduction in professional autonomy

- The professions can make a positive use of the consent process and EBT, in order to regain public trust. awarded by the courts. Criticism also comes from within the profession's own ranks. In a recent article entitled 'How Doctors betrayed us all' the editor of the Lancet called into question the whole ethos of professional autonomy by describing the medical establishment as being unnecessarily 'secretive and unaccountable', prompted by the reluctance of some professionals to expose colleagues that had been practising outside of both ethical and legal parameters. ${ }^{5}$

It would be all too easy to view the growing number of medical negligence cases with trepidation instead of viewing them with an educational eye, and learning from mistakes made by some professionals caught up in the net of litigation suits. What has emerged from many of the cases, is that some of the basic principles of patient autonomy such as 'consent' are not always that clearly defined and therefore not always that clearly understood by many professionals. This concern has been recently echoed in the literature by junior doctors and others. ${ }^{6-8}$

The issue of consent is only the starting point from an educational perspective. The current push for evidence-based treatment (EBT) inevitably impacts on the issue of consent and may differ when viewed from either a therapeutic or non-therapeutic focus. This may leave an area of uncertainty in which some professionals can be challenged when making decisions with their patients. Clearly both the concept of consent and the growing demand for EBT must be accepted by the professions if they are to avoid any further diminution to their autonomy.

Some professionals may not yet be familiar with the concepts of evidence-based treatment and the correlation between the latter and consent. There are a growing number of healthcare professionals who have taken on board the positive aspects of acknowledging patient participation in the decision making process. A recent editorial in the BDJ stated: ${ }^{9}$

'the questioning by a patient of the treatment offered to him is a reason to welcome evidence-based treatment . . . a dentist should be in a position to respond with good quality information that is backed by appropriate 
references' and 'this in turn will improve the quality of informed consent'.

Informed consent may be a transatlantic construct but, like many other social constructs, after a period of socialisation the concept assumes a reality of its own that we all accept and therefore live by.

The aim of this paper is to investigate the effect of evidence-based treatment on the legal requirements of consent, when the treatment transcends the therapeutic boundary and as a consequence any potential effects on the autonomy of healthcare professionals.

\section{Consent}

The climate of change now being experienced by many professionals in the healthcare system means that for almost all medical treatment procedures implied consent' may offer limited defence when even minor procedures go wrong. The very nature of consent may prove to be problematic for many when moving from the conceptual to the practical, especially for the inexperienced. Some of the problems associated with the obtaining of consent have been stated to be: ${ }^{6-8,10}$

- Lack of relevant knowledge on the part of the health professional

- How much information to give to the patient in a specific situation

- Level of understanding on the part of the patient

- Ability of the patient to comprehend the information given to them.

In the past the trust ascribed to health professionals by their patients made the acquiring of consent a formality. Rowe in 1994 describes implied consent and wrote: ${ }^{10}$

'by being in the chair at the dental surgery with mouths open a patient implies that they are there for dental treatment' and continued 'in the past a dentist would undertake treatment as he or she saw fit, which the patient would accept without argument'.

In recent years such absolute clinical freedom has been eroded due in part to what Eve and Hodgkin have described as steady growth in patients' right culture, economic and re-organisational changes. ${ }^{11}$

The patients' rights culture has perhaps been the most far reaching of the changes that have fed both public and legal interest, which has in turn, led to a re-examination of patient consent and professional autonomy when making decisions about patients medical treatment.

In the sphere of therapeutic treatment consent is said: ${ }^{11}$

'not to be valid unless the patient understands the nature and purpose of the treatment. Once a person is informed in broad terms of the nature of the procedure and gives consent, the consent is valid. However in circumstances where consent is induced by deceit or given under a misapprehension as to the nature or purpose of the treatment consent cannot be said to be valid. More uncertainty surrounds the situation where the patient is aware of the nature and purpose of the treatment but unaware of its harmful consequences. Where the harmful consequences are not reasonably foreseeable the professional may be able to rely on the consent given by the patient'.

The problem of obtaining consent from a minor may be even more problematic even if the minor is adjudged to be legally competent, ${ }^{12}$ and is outlined in Figure 1.

Current advice given to some healthcare professionals by their defence organisation is to provide the patient with full and frank disclosure of all the benefits and all the risks.
There is a current body of legal opinion that suggests that although consent remains vital to any legal defence in a medical negligence claim, consent alone will not shield a professional from litigation because the courts may hold that a patient cannot consent to negligent treatment. ${ }^{13}$ This reflects some of the main principles of EBT.

In the sphere of 'non-therapeutic treatment' consent is primarily defined by the Declaration of Helsinki which states that the subject must be informed of all the benefits and all the risks. ${ }^{14}$ Leaving little scope for the defences for non-disclosure for purely therapeutic procedures. As Mason and McCall state: ${ }^{15}$

"Resort to innovative technique may be appropriate in certain cases but should be made with caution. Whether or not the use of such a technique could amount to negligence would depend on the extent to which its use was considered justified in the case in question. In assessing this, a court would consider evidence of previous trials of the treatment and would also, no doubt, take into consideration any dangers, which it entailed. It is possible that a court would decline to endorse the use of an untried procedure if the patient was thereby exposed to considerable risk of damage. Other factors, which might be taken into account, would be the previous response of the patient to more conventional treatment, the seriousness of the patient's condition and the attitude of the patient himself towards the novelty and risk. The standard of care to be
Fig. 1 Potential participants in child consent

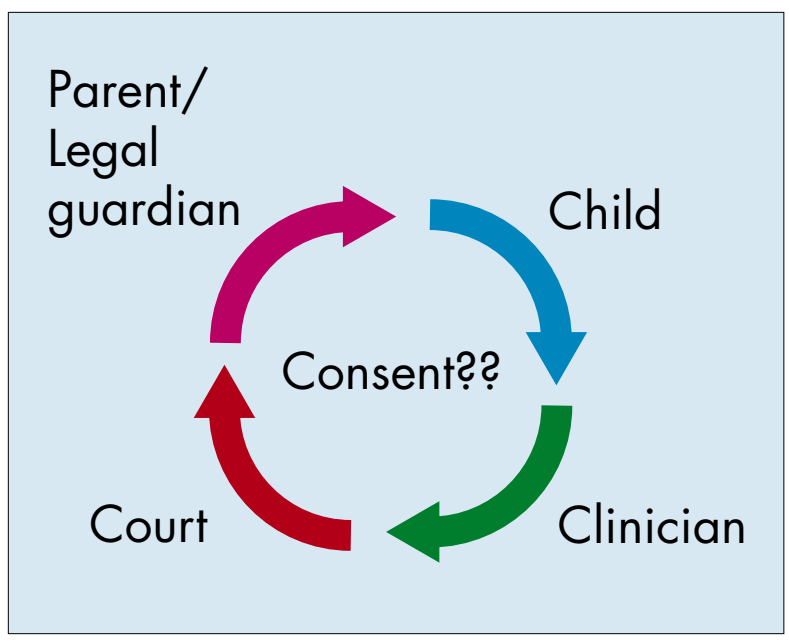


applied in such circumstances would be expected of a doctor who is reasonably competent in the provision of such treatment. A doctor should not, therefore, undertake procedures which are beyond his/her capacity'.

In many medical negligence claims the issue of consent has been a prominent feature examined by the judiciary. But up until now the legitimacy of the consent obtained by some health care professionals when operating in the grey area between therapeutic and non-therapeutic treatment procedures has received scant attention by the British courts. Many potential claims are settled out of court and therefore rarely receive the amount of media coverage and thus public scrutiny that strictly therapeutic cases receive. The current push for EBT has opened a potential forum for debate on the issue of consent, in recognition of the changing economic, political and social pressures that impact on the healthcare system in general. Moreover, the establishment of the purchaser/provider structure within the healthcare provision has resulted in a separation of managerial powers not previously experienced by the healthcare professions, adding additional stakeholders in the debate, which are listed in Figure 2.

\section{Evidence-based treatment}

The term evidence-based treatment has been described as 'consistent and judicious use of best evidence available when making decisions with patients.', 16 This definition has to be interpreted widely as EBT is an objective approach to patient treatment based on updated knowledge which requires, inter alia, that the professional carrying out the treatment has the appropriate level of competencies and required skill to undertake the treatment or procedure.

Evidence-based treatment may not be a concept that every health professional is familiar with. However the present economic, social and political changes necessarily demand that EBT principles will be implemented. The rate of implementation may be dependent on the number of health procedures that have been submitted to strict EBT criteria. For example, dentistry claims only a limited number of dental health procedures as fulfilling the strict cri-

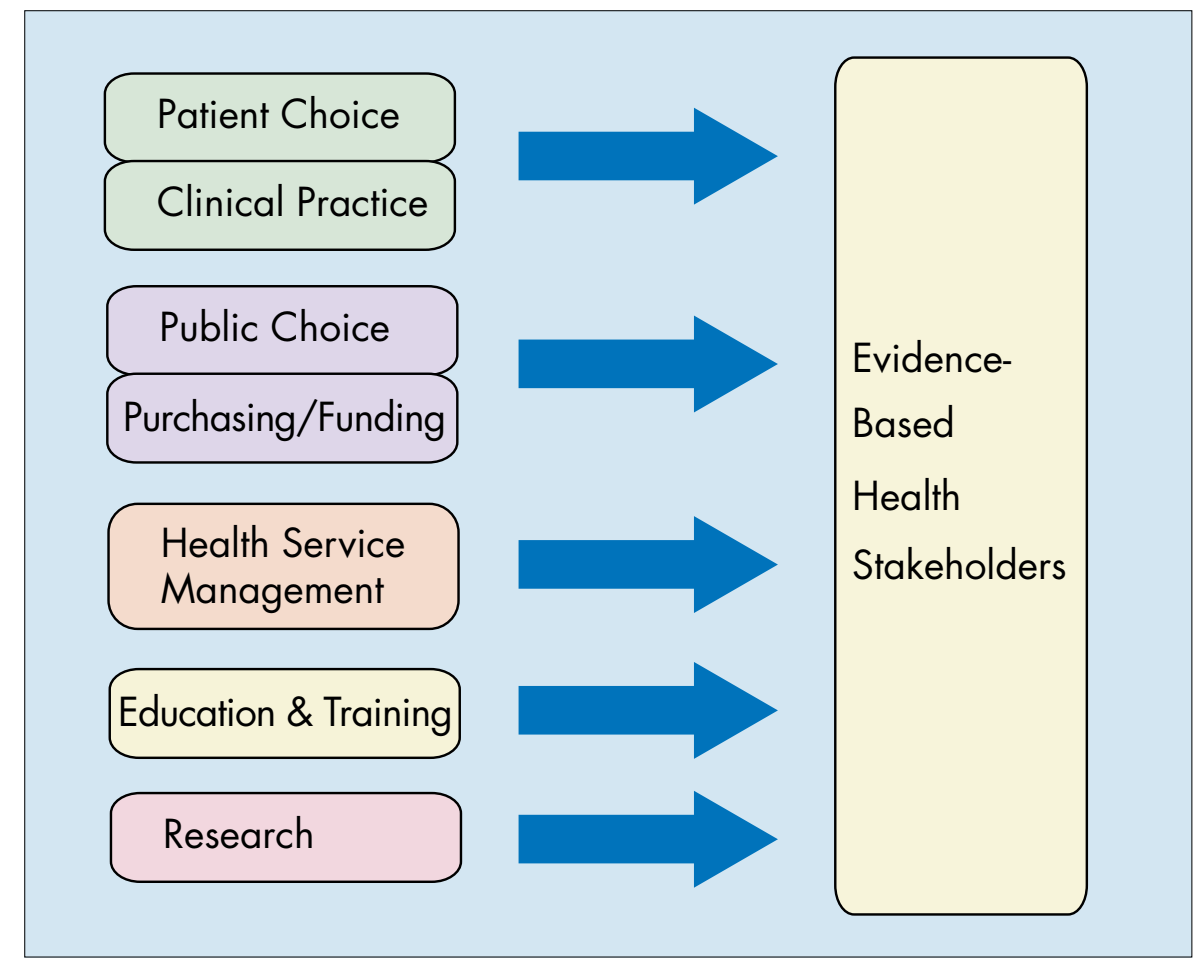

Fig.2 Stakeholders in evidence-based treatment

teria of EBT principles, instead basing most dental health procedures on the less rigorous criteria of treatment based on evidence.

Article 129 of the Treaty of Rome (as amended at Maastrict) gave new powers to the EU institution to develop and implement Public Health programmes at EU level. ${ }^{17}$ At a macro level the committee's findings suggested that if health policies are to be successful they should be based upon:

- Health needs assessment

- That such assessments should be evidence based.

At a micro level increasing prominence is to be given to the use of best current evidence when making decisions with patients encapsulating:

- Clinical practice

- Health care management

- Purchasing.

The intended or unintended consequences of the current push for EBT, at both a macro and micro level, has the potential to reforge the healthcare system, in that it would require not only a state-of-the-art knowledge on the part of the professional, but also the way such knowledge is selected and applied to both public and private health issues, for patients collectively and individually. Although the term 'evidencebased' may apply to many other disciplines and professions, the focus in the UK is on the healthcare system primarily because of the increasing economic pressures in servicing a growing population with a decreasing budget. This above all else appears to have been the main impetus of the current interest in EBT.

Eve and Hodkin suggest EBT is not a new concept and have outlined other important factors that have provided justification for its implementation: ${ }^{11}$

- Medical practice variation

- Rise in litigation

- Patient rights' culture

- Internal market in healthcare.

\section{Stakeholders}

Gray associates EBT with activities and he writes 'the term "evidence-based health- 
care" embraces a wide variety of activities, each of which should be evidence based. ${ }^{16}$ Gray understates the impact of EBT in the current stakeholder society. The stakeholders identified in Figure 2 all have a vested interest and the associated EBT activity may cause conflict by requiring differing levels of accountability and as a consequence encroach on the perceived and actual autonomy of each group of stakeholders, including patient choice which may be limited by resources and available treatments. ${ }^{18}$

\section{Autonomy}

The patient and the health professional must be central to any discussion on autonomy within a health provision setting, but as already indicated other stakeholders have a vested interest and will have varying degrees of influence on levels of both patient and professional autonomy. This is exemplified by Mason and McCall who state 'autonomy must be qualified by the legitimate interest and expectations of others, as well as economic constraints.' ${ }^{15}$

Patient autonomy within healthcare provision will always be subject to political, economic and judicial constraints and may increasingly come under the control of the health managers. The principles of EBT may apply similar constraints on professional autonomy, because it is the professionals who assume ultimate responsibility for patient treatment, particularly when the treatment goes wrong and results in consequential damage. The current climate of change not only impacts on patient choice, but necessarily redraws the boundaries between patient and professional autonomy, facilitated by the push for EBT.

Professional autonomy can be separated into institutional and individual autonomy; both rely on a perceived and actual trust enshrined in the professional practice motto of 'credat emptor'. Professionals within the healthcare system have a long history of autonomy, premised on claims of superior knowledge and objective scientific truth. This autonomy is now being questioned as a result of the action of some individuals within the profession. Recent events like the case of Wisheart have had a negative impact on public perception and as a consequence there has been a loss of public trust in the health professions. $^{3,4}$

Such cases has been compounded by other medical discrepancies such as widespread practice variation, described by Eve and Hodkin where similar patients are treated for the same diagnosis have hugely variable outcomes depending on the clinician, hospital and geographical location, which to date is unexplained in any real terms. ${ }^{11}$ The latter may pose the greatest challenge to the autonomy of health professionals, not because practice variation is peculiar to the healthcare system per se but because, as Eve and Hodkin assert, they make larger claims than most premised on objective scientific truth. ${ }^{11}$

Medical practice variation, combined with:

- Rising costs of healthcare

- Creation of internal market

- Consumerism and the consequent rise in litigation and payouts awarded by the courts (both estimated at around $15 \%$ by the Medical Defence Union)

- The information revolution, facilitated by free movement of information as a result of the technological advances in electronic communication

The above points have led to both the European Union and national interest promoting EBT as part of a risk management strategy at the macro and micro level.

At a national level practice variation has allowed professional autonomy to come under the scrutiny of the controlling bodies of the professions, third party insurers and the many NHS Trusts, who pick up the bill for litigation costs when mistakes are made. It has also come under the gaze of the general public who are becoming increasingly more litigious when treatment or procedures go wrong or transcend the therapeutic boundary.

A distinction between therapeutic and non-therapeutic treatment has been made by Capron who defines the former as 'treatment, which is an established treatment or procedure with a known beneficial outcome. ${ }^{19}$ He defined non-therapeutic treatment as 'the use of innovative treatment or procedures that have no established outcome'. The distinction enables the authors to highlight the link, shown in Figure 3, between EBT and therapeutic procedures

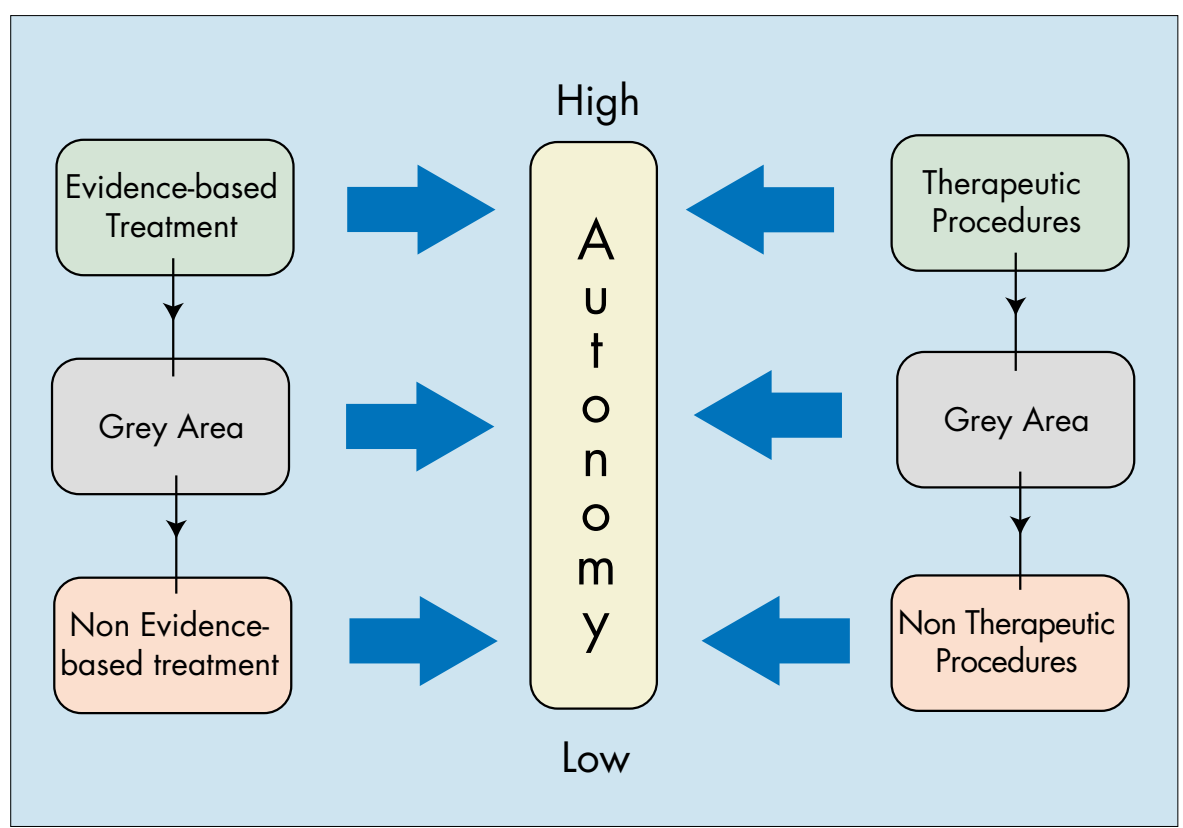

Fig. 3 Influences on professioal autonomy 
showing that there will be a grey area in between, where the distinction may lack clarity and therefore be open to legal challenge and ethical disputes.

\section{Legal implications}

Some of the possible legal implications of obtaining the patients consent to both therapeutic and non-therapeutic treatment have already been stated succinctly by Mason and McCall. ${ }^{15}$ The effect of EBT on the process of consent is still relatively untested particularly in the grey area of treatment. Yet recent cases suggest that EBT will impact on the decision-making process where treatment is concerned and, as a consequence, has the potential to significantly narrow the grey area between therapeutic and non-therapeutic treatment.

Therapeutic procedures, identified by EBT principles based on informed consent; carried out by skilled and competent professionals, offers a way to minimise unnecessary claims of medical negligence.

Resort to non-therapeutic treatment that transcends the therapeutic boundary, and can therefore be argued to be essentially non-evidenced-based treatment, may be appropriate in certain cases, but should only be undertaken with caution and then only with the patient's expressed written consent.

\section{Discussion}

Professions and professionals have a perceived autonomy vested in their claim to objective scientific truth, which promotes public trust. Events like the Wisheart case fuelled by media exposure, have resulted in a loss of public trust in the health professions. ${ }^{3,4}$ Health professionals need to be seen to carry out best practice procedures based on EBT to regain public trust.

Patients have the legal right to decide what may or may not be done to them, enshrined in the classic expression by Cardozo: ${ }^{20}$

'Every human being of adult years and sound mind has a right to determine what shall be done to his own body; and a surgeon who performs an operation with- out the patient's consent commits an assault'.

Consent has long been a contentious issue in many claims of medical negligence. EBT, even if interpreted narrowly, adds to the consent debate because of the additional requirements embodied in its main principles.

The review revealed that although consent remains the cornerstone of defence in many areas of therapeutic treatment, it is open to significant legal challenge in the grey area between therapeutic EBT and non-evidence based procedures. The former usually requires sufficient information to be given to or on behalf of the patient, whereas the latter demands a much higher level of information and understanding on the part of the decisionmaker. Establishing what constitutes therapeutic treatment from non-therapeutic procedures offers ample opportunity for legal dispute. EBT significantly narrows this grey area of treatment and may offer a way to minimise legal disputes and regain public trust.

\section{Conclusion}

A loss of patient trust in the health professions has resulted in a rise in litigation and payouts by the courts. Consent remains the cornerstone of defence for therapeutic procedures. It is less certain with innovative treatment or procedures that transcend the therapeutic boundaries and are shown to be non-EBT. The practice of EBT may give additional protection to health professionals, while addressing the vested interests of the other stakeholders. The rationale behind EBT necessarily impacts on the current debate on professional autonomy, and by implication raises questions on accountability and continued self-governance of many professions.

It can be argued that consent and EBT have become the tool of litigants, with a reduction in professional autonomy. However, in the redrawing of the boundaries, the professions can make a positive use of the consent process and EBT, in order to regain public trust, and at the same time address one of the major criticisms voiced by consumers of healthcare services which is, to improve compliance by implementing clearly defined professional codes.

1 G Cannavina, A Johnson, R B Winstanley. Lifelong Education and the Dental Profession. Eur J Prosthodont Rest Dent 1998; 6: $2 ; 51-54$.

2 Hughes E C. Professions daedalus fall. pp655669. New York: Free-Press, 1963.

3 Dunn P M. The Wisheart: paediatric cardiological services in Bristol, 1990-5. BMJ 1998; 317: 1144-1155.

4 Bolsin S N. The Wisheart affair: responses to Dunn. BMJ 1998; 317: 1579-1580.

5 Horton R. How Doctors have betrayed us all. Independent on Sunday 1998; June: 11.

6 Hutchinson M. Consent review steps up a gear. Hospital Doctor 1998; July: 4.

7 Johnson P. Why consent issue must be dealt with. Hospital Doctor 1998; July: 16.

8 Mulcahy D et al. Informed consent from whom? J R Coll Surg Edinb 1997; 42: 161-164.

9 Lawrence A. Welcome to evidence based dentistry. BDJ 1998; 1: 2 .

10 Rowe A H R. Consent. Dent Update 1994; June: 188-190.

11 Eve R, Hodgkin P. Professionalism and medicine. In Broadbent J, Dietrich M, Roberts $\mathrm{J}$ (eds). The end of the professions? pp69-84. London: Routledge, 1997.

12 Cannavina C D. The use of children in medical experimentation. MA Dissertation; The University of Sheffield, 1997.

13 Sfikas P M. Informed consent and the law. JADA 1998; 129: 1613-1614.

14 World Medical Association Declaration Helsinki: Recommendations Guiding Physicians in Biomedical Research Involvement. JAMA 1997; 277: 925-926.

15 Mason J K, McCall R A. Law and Medical Ethics. pp 218-229. London: Butterworths, 1994.

16 Gray J A M. Evidence based public health what level of competencies required. J Public Health Med 1997; 19: 65-68.

17 European Communities No. 3 Treaty as European Union including the Protocol and Final Act with Declaration, Maastricht, 7 February 1992. London: HMSO, 1992.

18 C D Cannavina, G Cannavina, D Longley. The legal status of a child's consent to treatment. $J$ Dent Res 1998; 77: Issue B, Abst 1579.

19 Capron A. In McNeill P. The Ethics and Politics of Human Experimentation. pp 126. Cambridge Press, 1993.

20 Cardozo J. In Mason J K, McCall R A. Law and Medical Ethics. pp 219. London:Butterworths, 1994. 